\title{
P216
}

\section{A Comparison of Continuous Mass-lumped Finite Elements and Finite Differences for 3D}

\author{
E. Zhebel* (Shell Global Solutions International BV), S. Minisini (Shell \\ Global Solutions International BV), A. Kononov (Source Contracting) \& W. \\ A. Mulder (Shell Global Solutions International BV / TU Delft)
}

\section{SUMMARY}

The finite-difference method is widely used for time-domain modelling of the wave equation because of its ease of implementation of high-order spatial discretization schemes, parallelization and computational

efficiency. However, finite elements on tetrahedral meshes are more accurate in complex geometries near sharp interfaces. We compared the fourth-order finite-difference method to fourth-order continuous masslumped finite elements in terms of accuracy and computational cost. The results show that for simple models like a cube with constant density and velocity, the finite-difference method outperforms the finite-element method by at least an order of magnitude. For a model with interior complexity and topography, however, the finite elements are about two orders of magnitude faster than finite differences. 


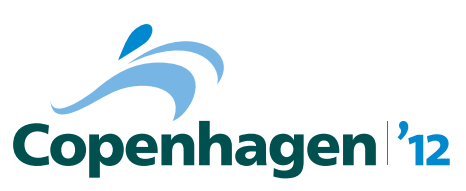

\section{Introduction}

The finite-difference method has become the workhorse for time-domain modelling of the wave equation with applications in acquisition optimization, development and testing of seismic processing algorithms, reverse-time migration and full waveform inversion. Its advantages are the relative ease of coding and parallelization, the use of high-order spatial discretization schemes and its computational speed. Unfortunately, the method suffers from loss of accuracy near sharp interfaces. If the interface does not follow the grid, the staircasing effect generates local first-order errors. Because the solution is continuous but not differentiable across an impedance contrast, a local second-order error will be incurred as well. Finite elements that follow sharp interfaces do not suffer from this loss of accuracy. Continuous masslumped finite elements allow for explicit time stepping at the expense of requiring additional degrees of freedom to maintain their spatial accuracy.

After the presentation by Lesage et al. (2010) at the EAGE meeting in Barcelona, the question was asked how the continuous mass-lumped finite elements of degree 2 compare to the finite-difference method. In 2D, the answer is known (Mulder, 1996): higher-order mass-lumped finite elements outperform the finite-difference method in terms of the computational time required to obtain a solution with a given numerical accuracy as soon as the velocity model has some non-trivial structure. In spite of the additional complexity of the finite elements, the use of larger triangles where the velocity is higher allows them to be more efficient than finite differences. In 3D, it is less obvious if finite elements are superior in efficiency. The 3-D mass-lumped tetrahedral elements have a relatively large number of nodes compared to the standard element (Mulder, 1996; Chin-Joe-Kong et al., 1999). To answer the question of efficiency, we consider the worst-case example of topography.

\section{Method}

We solve the acoustic wave equation

$$
\frac{1}{\rho c^{2}} \frac{\partial^{2} p}{\partial t^{2}}-\nabla \cdot \frac{1}{\rho} \nabla p=f,
$$

in $3 \mathrm{D}$, using the finite-difference (FD) or continuous finite-element (FE) method. Here, $p(t, \mathbf{x})$ is the pressure as a function of time $t$ and position $\mathbf{x}=(x, y, z)$, whereas $c(\mathbf{x})$ is the wave velocity and $\rho(\mathbf{x})$ the density. The source term $f(t, \mathbf{x})=w(t) s\left(\mathbf{x}-\mathbf{x}_{s}\right)$, with wavelet $w(t)$ and spatial source function $s$, typically a delta function that peaks at the source position $\mathbf{x}_{s}$.

For the finite-element method, we consider mass-lumped continuous elements with polynomial basis functions. On triangles in 2D, they have to be enriched with polynomials of higher degree in the interior to avoid loss of accuracy after lumping (Fried and Malkus, 1975). At present, elements are known for degrees 1 to 6 (Fried and Malkus, 1975; Tordjman, 1995; Cohen et al., 1995, 2001; Mulder, 1996; ChinJoe-Kong et al., 1999; Mulder, 2011). The 3-D extension to the tetrahedron (Mulder, 1996) required higher-degree polynomials in the interior of the faces and of the tetrahedron and resulted in an element of degree 2 on the edges and 4 in the interior of faces and element. Chin-Joe-Kong et al. (1999) found 2 types of elements with degree 3 on the edges. Lesage et al. (2010) and Zhebel et al. (2011) presented 3-D applications of these elements.

For our comparison, we used an acoustic FD code (Mulder, 1997). The source was represented as a tapered sinc, a variant of the one proposed by Hicks (2002). For the mass-lumped finite elements, we only considered the augmented element of degree 3, type 2 (Chin-Joe-Kong et al., 1999), which has a more favourable time-stepping stability limit than type 1 (Zhebel et al., 2011). The element is enriched with polynomials of degree 5 on the interior of the faces and of degree 6 in the interior of the tetrahedron, leading to a total of 50 nodes for each element compared to 20 for the standard element. The third-degree augmented finite element and the fourth-order finite differences have both formally a fourth-order spatial error. 


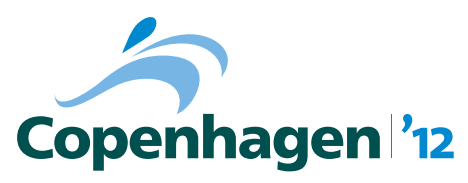

To simulate topography in the finite-difference code, we took the approach of Bartel et al. (2000). We added an extra layer on top of the computational domain, taking the velocity of the top layer of the solid model, but a low density of $0.0013 \mathrm{~g} / \mathrm{cm}^{3}$. In the solid part of the model, the density was set to $1 \mathrm{~g} / \mathrm{cm}^{3}$. To avoid instabilities, an extra point with a density of $0.1 \mathrm{~g} / \mathrm{cm}^{3}$ was added just above the free surface, as recommended by Bartel et al. (2000). We prefered to simulate the topography with a density contrast rather than with a velocity contrast to avoid air wave artefacts in the seismic data. The disadvantage of the large density contrast is a reduction of the Courant-Friedrichs-Lewy (CFL) stability bound (Courant et al., 1928) below the usual von Neumann stability estimate.

We need to have an estimate of the numerical errors produced by the codes as a function of the elapsed time. Since the exact solution is unknown, the absolute error can be estimated from the relative errors, obtained by comparing the differences between solutions on a sequence of grids of various mesh sizes. Wang et al. (2010) presented an example of this approach in a geophysical context. Let $h$ be a measure of the grid spacing. Instead of the spacing, we take $h=N^{-1 / 3}$, where $N$ is the number of grid points of the finite-difference mesh or the number of degrees of freedom of the finite-element discretization. We expect the spatial error to behave as some power $q$ of $h: p^{h}-p_{\text {exact }}^{h} \simeq a h^{q}$, where $a$ is a constant depending on position and time, $p^{h}$ is the numerical solution on a given mesh and $p_{\text {exact }}^{h}$ the projection of the exact solution to the same mesh. The relative error on two meshes with $h_{1}$ and $h_{2}$ will then behave as $\left\|p^{h_{1}}-p^{h_{2}}\right\| \simeq\|a\|\left|h_{1}^{q}-h_{2}^{q}\right|$. For the norm, we will consider the maximum $\left(L_{\infty}\right)$ norm and the standard quadratic $\left(L_{2}\right)$ or 2-norm. Given runs on three different grids, we can estimate $\|a\|$ and $q$ with a nonlinear fit, assuming the spatial error dominates over the time stepping error. Since the error behaviour is only an asymptotic estimate, additional runs may be useful to assess the quality of the result.

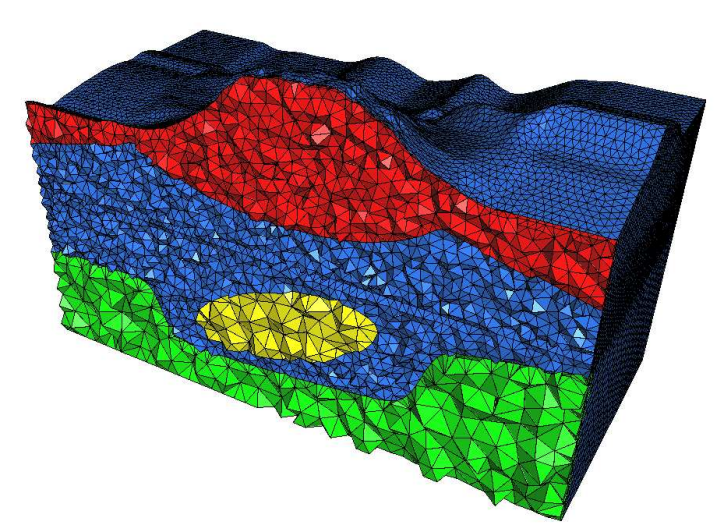

Figure 1 Section through the coarsest tetrahedral mesh.

\section{Results}

Figure 1 depicts a section through the coarsest tetrahedral mesh used in the FE computations. The finite-element mesh was generated in such a way that the element size scaled with velocity. Figure 2 shows a vertical cross-section of the somewhat unrealistic velocity model with the FE wave field after $0.5 \mathrm{~s}$ superimposed. A shot was placed in the centre of the model, at $x_{s}=2000 \mathrm{~m}, y_{s}=2000 \mathrm{~m}$, and $10 \mathrm{~m}$ below the surface. Receivers where placed from $x_{r}=1012.5 \mathrm{~m}$ through $2987.5 \mathrm{~m}$ at a $25-\mathrm{m}$ interval, $y_{r}=$ $2000 \mathrm{~m}$ and at $10-\mathrm{m}$ depth below the surface in the vertical direction. Figure 3 displays a seismogram computed with the FE method.

Figure 4(a) shows estimates of the absolute error, $\|a\| h^{q}$, in the trace data for FD and FE as a function of the degrees of freedom $N$ to the power 1/3, using 3 runs for each. Although the FD method had formally a fourth-order spatial error, but an estimated error somewhere between first- and second-order, as expected. The estimated power for the error in the FE method is better than 5, but was set to its theoretical value of 4, giving less optimistic estimates. Still, Figure 4(b) demonstrates that the efficiency 


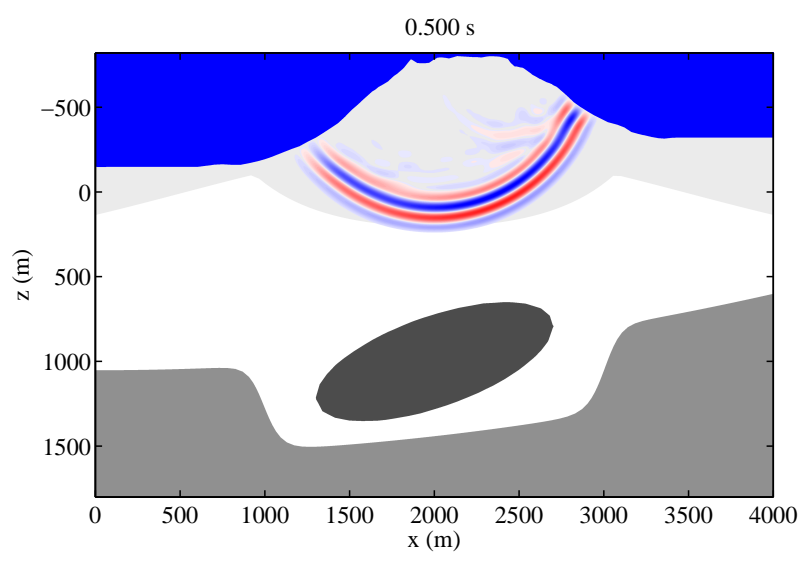

Figure 2 Vertical cross section at $y=2 \mathrm{~km}$ after 0.5 seconds. Positive pressure values are shown in red, negative in blue. The backdrop represents the velocity model on a gray scale, with values of $1.8,1.5,3.0$ and $3.9 \mathrm{~km} / \mathrm{s}$ from light to dark. The sky is blue.

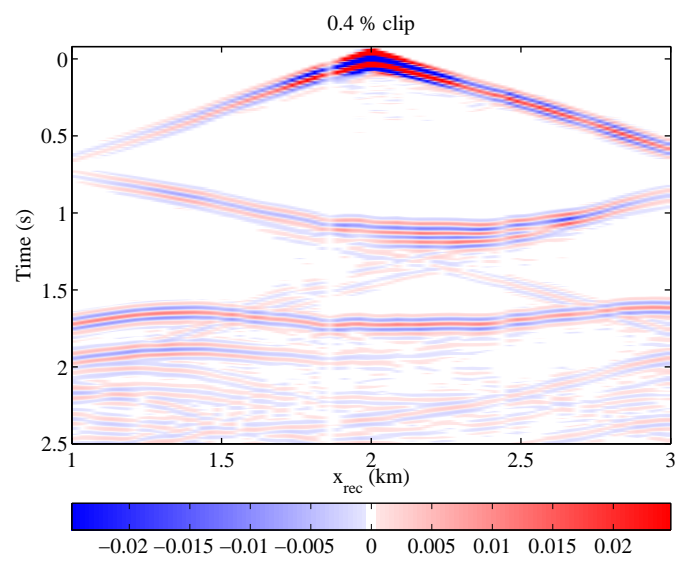

Figure 3 Data for a shot at $x=2$ and $y=2 \mathrm{~km}$. Shots and receivers are buried $10 \mathrm{~m}$ below the surface.

of the finite-element method is superior. To achieve, for instance, an accuracy of $1 \%$, the FE method is about 2 orders of magnitude faster than FD. Results were obtained on 12 cores on a single board, using MPI for the FD code and OpenMP with multi-threading for the FE code.

At the other extreme, a cube with constant velocity and density, FD beats FE by at least one order of magnitude in speed.

Of course, the timings depend on implementation details, code optimization, compilers and hardware. It should be mentioned that FE uses local reassembly of the stiffness matrices at each time step to save storage. We have observed that global assembly will speed up the code by a factor of about 3 , far less then can be expected by looking at the number of floating point operations. This is due to memory access and cache misses. We want to emphasize that the present results should be taken only as crude indications of performance.

\section{Conclusions}

We have compared a fourth-order continuous finite-element method to a standard fourth-order finitedifference method in terms of computational efficiency, meaning the elapsed computational time it takes to obtain a numerical solution at fixed accuracy. For a model with constant velocity, the finite-element method is inefficient compared to finite differences. At the other extreme, a model with sharp interfaces and topography, this particular type of finite element easily wins.

\section{References}

Bartel, L.C., Symons, N.P. and Aldridge, D.F. [2000] Graded boundary simulation of air/Earth interfaces in finite-difference elastic wave modeling. SEG Technical Program Expanded Abstracts, 19(1), 2444-2447, doi: 10.1190/1.1815958.

Chin-Joe-Kong, M.J.S., Mulder, W.A. and van Veldhuizen, M. [1999] Higher-order triangular and tetrahedral finite elements with mass lumping for solving the wave equation. Journal of Engineering Mathematics, 35(4), 405-426, doi:10.1023/A:1004420829610.

Cohen, G., Joly, P., Roberts, J.E. and Tordjman, N. [2001] Higher order triangular finite elements with mass lumping for the wave equation. SIAM Journal on Numerical Analysis, 38(6), 2047-2078, doi: $10.1137 /$ S0036142997329554.

Cohen, G., Joly, P. and Tordjman, N. [1995] Higher order triangular finite elements with mass lumping for the wave equation. Proceedings of the Third International Conference on Mathematical and Numerical Aspects of Wave Propagation, SIAM, Philadelphia, 270-279. 


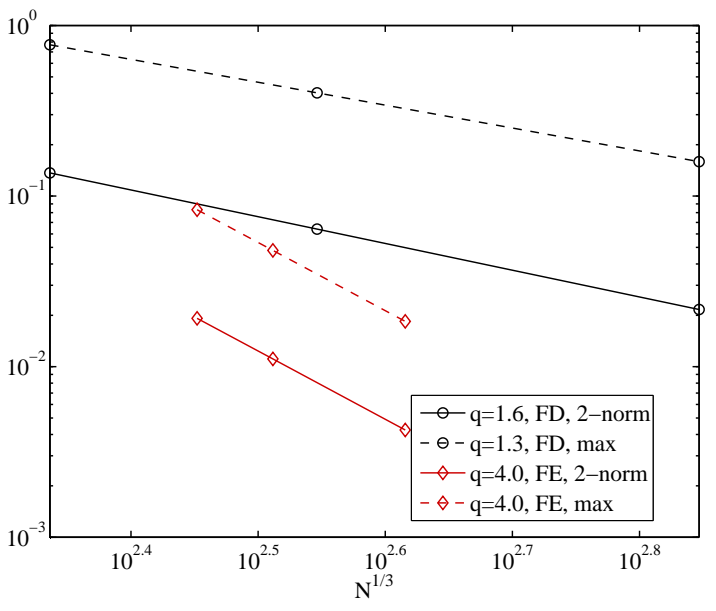

(a)

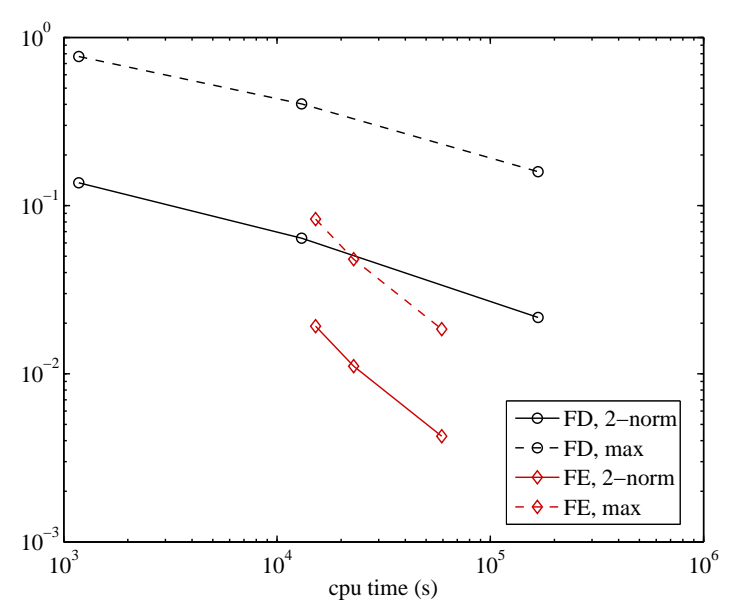

(b)

Figure 4 (a) Estimated error as a function of $1 / h=N^{1 / 3}$, where is $N$ is the number of grid points for the finite-difference method $(F D)$ or the number of degrees of freedom for the finite-element method $(F E)$. The dashed lines represent the maximum norm, the drawn the 2-norm. (b) Estimated error as a function of computational time on 12 cores.

Courant, R., Friedrichs, K. and Lewy, H. [1928] Über die partiellen Differenzengleichungen der mathematischen Physik. Mathematische Annalen, 100(1), 32-74, doi:10.1007/BF01448839.

Fried, I. and Malkus, D.S. [1975] Finite element mass matrix lumping by numerical integration with no convergence rate loss. International Journal of Solids and Structures, 11, 461-466.

Hicks, G.J. [2002] Arbitrary source and receiver positioning in finite-difference schemes using Kaiser windowed sinc functions. Geophysics, 67(1), 156-165, doi:10.1190/1.1451454.

Lesage, A.C., Aubry, R., Houzeaux, G., Polo, M.A. and Cela, J.M. [2010] 3D spectral element method combined with H-refinement. 72nd EAGE Conference \& Exhibition, Barcelona, Spain, Extended Abstracts.

Mulder, W.A. [1996] A comparison between higher-order finite elements and finite differences for solving the wave equation. Proceedings of the Second ECCOMAS Conference on Numerical Methods in Engineering, John Wiley \& Sons, Chichester, 344-350.

Mulder, W.A. [1997] Experiments with Higdon's absorbing boundary conditions for a number of wave equations. Computational Geosciences, 1(1), 85-108, doi:10.1023/A:1011556926362.

Mulder, W.A. [2011] New triangular mass-lumped finite elements of degree 6 for wave propagation. Applied Numerical Mathematics, submitted.

Tordjman, N. [1995] Élements finis d'order élevé avec condensation de masse pour l'equation des ondes. Ph.D. thesis, L'Université Paris IX Dauphine.

Wang, X., Symes, W.W. and Warburton, T. [2010] Comparison of discontinuous Galerkin and finite difference methods for time domain acoustics. SEG Technical Program Expanded Abstracts, 29(1), 3060-3065, doi: 10.1190/1.3513482.

Zhebel, E., Minisini, S., Kononov, A. and Mulder, W.A. [2011] Solving the 3D acoustic wave equation with higher-order mass-lumped tetrahedral finite elements. 73rd EAGE Conference \& Exhibition, Vienna, Austria, Extended Abstracts. 